\title{
Computing the Enclosures Eigenvalues Using the Quadratic Method
}

\author{
Shurouq Mohammad Abusamra \\ Department of Mathematics, Faculty of Science, King Abdul-Aziz University, Jeddah, KSA \\ Email: ashurouq@yahoo.com
}

How to cite this paper: Abusamra, S.M. (2019) Computing the Enclosures Eigenvalues Using the Quadratic Method. $A p$ plied Mathematics, 10, 212-225. https://doi.org/10.4236/am.2019.104016

Received: February 7, 2019

Accepted: April 21, 2019

Published: April 24, 2019

Copyright (C) 2019 by author(s) and Scientific Research Publishing Inc. This work is licensed under the Creative Commons Attribution International License (CC BY 4.0).

http://creativecommons.org/licenses/by/4.0/

\begin{abstract}
In this article, We compute the enclosures eigenvalues (upper and lower bounds) using the quadratic method. The Schrodinger operator (A) (harmonic and anharmonic oscillator model) has used as an example. We study a new technique to get more accurate bounds. We compare our results with Boulton and Strauss method.
\end{abstract}

\section{Keywords}

Quadratic Method, Enclosures Eigenvalues, Boulton and Strauss Method

\section{Introduction}

Galerkin method is one of the best methods for determining upper bounds for the eigenvalues of semi-definite operators, unfortunately this method cannot find enclosures eigenvalue. This paper shows how to compute enclosures of the eigenvalues of self-adjoint operators by the Quadratic method. At first, we study the second-order relative spectrum (The Quadratic method) in [1] [2], and Boulton \& Strauss method in [3] [4]. These methods have used for computing eigenvalue enclosures (upper and lower bounds) of the eigenvalues of self-adjoin operators. The quadratic method, which relies on calculation of the second-order which is providing, certified a priori intervals of spectral enclosure. Then we study our new technique which gives more accurate results, we also follow the results that have been published by Boulton \& Hobiny in [5]. The method will be examined by harmonic and anharmonic oscillator models.

Second-order relative spectra were first considered by Davies (1998) in the context of resonances for general self-adjoint operators in [6] [7]. It was then suggested by Shargrodsky and subsequently by Levitin and Shargorodsky (2000) in [8] that the second order relative spectra can also be employed for the pollu- 
tion-free computation of eigenvalues in gaps of the essential spectrum. Various implementations, including on models from elasticity, solid state, physics, relativistic quantum mechanics and magneto hydrodynamics confirm that the Quadratic method is a reliable tool for eigenvalue approximation in the spectral pollution regime. Properties of second order relative spectra have been studied recently by Bolton \& Leviton in [9] and then by Bolton \& Strauss $(2007,2011)$ in [3] [4]. Boulton and Strauss extended this method to normal operators and optimal convergence rates for eigenvalues and estimated that by an order of magnitude for the harmonic \& anharmonic oscillator models, by cut the interval into sub-interval around the eigenvalues in the Spectrum, and the approximation enclosure eigenvalues results of these models are more accurate than the Quadratic method around $\lambda$.

Our improvement depends on domain expansion around the first five eigenvalues in the spectrum, we will take a value for $a$ less than $\lambda_{1}$ and a value for $b$ greater than $\lambda_{5}$ and calculate the conjugate pairs of eigenvalues $\left(\lambda_{1}, \lambda_{2}, \lambda_{3}, \lambda_{4}, \lambda_{5}\right)$ (i.e.; around $\lambda_{1}$ we will choose $a_{1}<\lambda_{1}<\lambda_{2}<\lambda_{3}<\lambda_{4}<\lambda_{5}<b_{1}$ ). In this paper we studied two models (harmonic and anharmonic oscillator) which are:

1): $\mathcal{H}^{\text {har }}(x)=-u^{\prime \prime}(x)+x^{2} u(x)$.

2): $\mathcal{H}^{\text {anh }}(x)=-u^{\prime \prime}(x)+x^{4} u(x)$.

\section{Notation}

- Below $\mathcal{H}$ denotes a generic separable Hilbert space with inner product $\langle$. and norm $\|\cdot\|$.

Let the operator $(\mathbb{A}: D(\mathbb{A}) \rightarrow \mathcal{H})$ be self-adjoint. We will write $\operatorname{Spec}(\mathbb{A})$ to denote the spectrum of $(\mathbb{A})$.

- $\mathcal{H}$ is real inner product space means that $\mathcal{H}$ is real vector space on which there is an inner product $\langle x, y\rangle$ associating a real number to each pair of elements $x, y$. The norm is real function such that $x=\langle x, x\rangle^{1 / 2}$, and the distance is: $d(x, y)=x-y=\langle x-y, x-y\rangle^{1 / 2}$. And:

$d(x, z) \leq d(x, y)+d(y, z) ; x, y \in \mathcal{H}$, which is called triangular inequality.

- For any pair of elements $x, y$ of $\mathcal{H}$ satisfies the following properties:

$$
\begin{gathered}
\langle x, x\rangle \geq 0 \\
\langle y, x\rangle=\langle x, y\rangle \\
\left\langle y, x_{1}+b x_{2}\right\rangle=\left\langle x_{1} y+b x_{2} y\right\rangle \\
\left\langle x, a y_{1}+b y_{2}\right\rangle=a\left\langle x, y_{1}\right\rangle+b\left\langle x, y_{2}\right\rangle
\end{gathered}
$$

- Given a subspaces $\mathcal{L} \subset D(\mathbb{A})$ of dimension $n$ such that:

$$
\mathcal{L}=\operatorname{Span}\left\{b_{j}\right\}_{j=1}^{n}
$$

we will write

$$
\mathbb{A}_{l}=\left[a^{l}\left(b_{j}, b_{k}\right)\right]_{k, j=1}^{n} \mathbb{C}^{n \times n}
$$

- The discrete spectrum: is the set of eigenvalues of finite multiplicity. 
- The essential spectrum of $\mathbb{A}:$ is the remaining part of $\operatorname{spec}(\mathbb{A})$.

- $\operatorname{Spec}_{\text {ess }}(\mathbb{A})=\frac{\operatorname{spec}(\mathbb{A})}{\operatorname{Spec}_{\text {disc }}(\mathbb{A})}$.

- Theorem: Let $\mathbb{A}$ be a self-adjoint operator:

- $\quad \lambda \in \operatorname{spec}(\mathbb{A})$, there exists a sequence $u_{j} \in \operatorname{Dom}(\mathbb{A})$ such that:

- $\left\|\lambda u_{j}, \mathbb{A} u_{j}\right\| \rightarrow 0$ as $j \rightarrow \infty$ [This sequence is called Welys sequence].

- $\lambda \in \operatorname{Specess}(\mathbb{A}) \leftrightarrow(\lambda-\mathbb{A})$ is not Fredholm.

- The min-max theorem tells us if $\mathcal{R}$ is bounded above and below by the mini-mum and maximum eigenvalues respectively $\left(\lambda_{\min }, \lambda_{\max }\right)$, and we have: $\lambda_{\min }$ (if any) $\leq \mathcal{R}(x) \leq \lambda_{\max }$ (if any), where $\mathcal{R}(x)$ denoted by Rayleigh-Ritz quotient on a self-adjoint operator $\mathbb{A}$ as:

$$
\mathcal{R}_{A(x)}=\frac{\langle\mathbb{A} x, x\rangle}{\langle x, x\rangle} ; x \in \operatorname{Dom}(\mathbb{A}) .
$$

\section{The Quadratic Method}

We begin by describing the basic framework of the Quadratic method associated to a self-adjoint operator. First considered by Davis (1998) in the context of resonances for general self-adjoint operator in [6] [7], it was then suggested by Shargrodsky and subsequently by Levitin and Shargorodsky (2000) in [8], Properties of second order relative spectra have been studied recently by Boulton \& Levitin in [9] and then by Boluton \& Strauss $(2007,2011)$ in [3] [4]. For the benefit of the reader, we include here some definitions.

Let $\mathbb{A}$ be self-adjoint operator on Hilbert space $\mathcal{H}$. Let $\mathcal{L} \subset \operatorname{Dom}(\mathbb{A})$, a number $z \in \mathbb{C}$ belongs to second order spectrum $\operatorname{Spec}_{2}(\mathbb{A}, \mathcal{L})$ if there exists $u, v \in \mathcal{L}$ such that;

$$
\langle(\mathbb{A}-z I) u,(\mathbb{A}-z I) v\rangle=0 .
$$

For all $u$ is nonzero. Means that the Second Order Spectrum usually contain complex numbers, but it turns out that if $z \in \operatorname{Spec}_{2}(\mathbb{A}, \mathcal{L})$ then: $\operatorname{Spec}(\mathbb{A})[(\operatorname{Re}(z)-|\operatorname{Im}(z)|),(\operatorname{Re}(z)+|\operatorname{Im}(z)|)] \neq \varphi$.

Consider: $\mathcal{E}=\left\{e_{1}, e_{2}, e_{n}\right\}$.

Be a basis of $\mathcal{L}[\mathcal{L}$ is finite dimensional subspace of $\operatorname{Dom}(\mathbb{A})]$, then the Quadratic matrix polynomial is:

$$
Q(z)=\mathbb{A}_{2}-2 z \mathbb{A}_{1}+z^{2} \mathbb{A}_{0} .
$$

where the mass, stiffness and bending matrices are;

$$
\left[\mathbb{A}_{0}\right]_{j k}=\left(e_{j}, e_{k}\right),\left[\mathbb{A}_{1}\right]_{j k}=\left(\mathbb{A} e_{j}, e_{k}\right),\left[\mathbb{A}_{2}\right]_{j k}=\left(\mathbb{A} e_{j}, \mathbb{A} e_{k}\right)
$$

We define the spectrum of $Q(z)$ as the set of $z \in \mathbb{C}$ such that

$$
Q(z)=0 \text { for some } u \in \mathcal{L} /\{0\} .
$$

The standard way of finding $\operatorname{Spec}(Q)$ is to reach to the linear pencil eigenvalue problem: 


$$
\mathbb{A} u=\lambda u \text { for some } u \in \mathcal{L} /\{0\} .
$$

In this method we need to construct companion matrices which depend on the quadratic matrix polynomial to find the enclosure eigenvalues.

We have two possible companion matrices form which are given by

$$
S=\left(\begin{array}{cc}
0 & I \\
-\mathbb{A}_{2} & 2 \mathbb{A}_{1}
\end{array}\right), \quad T=\left(\begin{array}{cc}
I & 0 \\
0 & \mathbb{A}_{0}
\end{array}\right)
$$

And

$$
S=\left(\begin{array}{rr}
0 & I \\
-\mathbb{A}_{2} & 0
\end{array}\right), T=\left(\begin{array}{cc}
I & 0 \\
2 \mathbb{A}_{1} & \mathbb{A}_{0}
\end{array}\right)
$$

The eigenvalues of the matrix polynomial can be determined from one of this companion matrices form

LEMMA 2. 1: Let $Q(z)$ is defined and be singular then:

$$
\operatorname{det}(Q(z))=0 \Leftrightarrow \operatorname{det}(S-z T)=0 .
$$

$$
\text { For } \begin{aligned}
S=\left(\begin{array}{cc}
0 & I \\
-\mathbb{A}_{2} & 2 \mathbb{A}_{1}
\end{array}\right), T=\left(\begin{array}{cc}
I & 0 \\
0 & \mathbb{A}_{0}
\end{array}\right) \\
\qquad \begin{aligned}
\operatorname{det}(S-z T) & =\left|\begin{array}{cc}
0-z I & I-0 \\
-\mathbb{A}_{2}-0 & 2 \mathbb{A}_{1}-z \mathbb{A}_{1}
\end{array}\right| \\
& =-z I\left(2 \mathbb{A}_{1}-z \mathbb{A}_{1}\right)+\mathbb{A}_{2} I \\
& =\operatorname{det}(Q(z))
\end{aligned}
\end{aligned}
$$

Indeed, the assertion that $Q(z)$ is singular is equivalent to the existence of $u \neq 0$ such that

$$
\mathbb{A}_{2} u-2 z \mathbb{A}_{1} u+z^{2} \mathbb{A}_{0} u=0 .
$$

Denoting $v=z u$, this can be rewritten as

$$
\mathbb{A}_{2} u-2 \mathbb{A}_{1} v+z \mathbb{A}_{0} v=0 .
$$

In turn, the latter is equivalent to:

$$
\left(\begin{array}{cc}
0 & I \\
-\mathbb{A}_{2} & 2 \mathbb{A}_{1}
\end{array}\right)\left(\begin{array}{l}
u \\
v
\end{array}\right)=z\left(\begin{array}{cc}
I & 0 \\
0 & \mathbb{A}_{0}
\end{array}\right)\left(\begin{array}{l}
u \\
v
\end{array}\right)
$$

As needed for the verification of (2.3).

LEMMA 2.2: Let $\mathcal{L}=\operatorname{Span}\left\{b_{j}\right\}_{j=1}^{n} \subset \operatorname{Dom}(\mathbb{A})$ and $\mathbb{P}$ is the orthogonal projection onto $\mathcal{L}$, then:

$$
\operatorname{Spec}_{2}(\mathbb{A} \mathcal{L})=\{z \in \mathbb{C} ? \exists u \in \mathcal{L} \quad\{\}((\mathbb{A}-z I) u(\mathbb{A}-z I) v)=\forall v \in \mathcal{L}\}
$$

If $\mathcal{L} \subset \operatorname{Dom}\left(\mathbb{A}^{2}\right)$, then:

$$
\begin{aligned}
\operatorname{Spec}_{2}(\mathbb{A} ; \mathcal{L}) & =\left\{z \in \mathbb{C}: \exists u \in \mathcal{L}-\{0\} ;\left\langle\mathbb{P}(\mathbb{A}-z)^{2} u, v\right\rangle=0, \forall v \in \mathcal{L}\right\} \\
& =\left\{z \in \mathbb{C}: 0 \in \operatorname{Spec}\left(\mathbb{P}(\mathbb{A}-z)^{2} \mid \mathcal{L}\right)\right\} .
\end{aligned}
$$

Typically $\operatorname{Spec}_{2}(\mathbb{A}, \mathcal{L})$ contains non-real points. From the lemma it is easy to see that $z \in \operatorname{Spec}_{2}(\mathbb{A}, \mathcal{L})$ if and only if $\bar{z} \in \operatorname{Spec}_{2}(\mathbb{A}, \mathcal{L})$. 
We now discuss a strategy suggested by Davis and Plum for computing the spectrum of self-adjoint operator.

Let $\mathbb{A}=\mathbb{A}^{*}$ and $\mathcal{L} \subset \operatorname{Dom}(\mathbb{A})$ for $z \in \mathbb{C}$ to $\operatorname{Spec}(\mathbb{A})$, consider the function $\mathbb{F}: \mathbb{C} \rightarrow[0, \infty)$ be given:

$$
\mathbb{F}(z)=\min _{v \in L} \frac{\|(z-\mathbb{A})\|}{\|v\|}
$$

Then $\mathbb{F}(z)$ is an upper bound for the distance from $z$ to the spectrum of $\mathbb{A}$, that means:

$$
\begin{gathered}
\operatorname{Dist}[z, \operatorname{Spec}(\mathbb{A})]=\min \{(z-\lambda): \lambda \in \operatorname{spec}(\mathbb{A})\} . \\
\text { So } \mathbb{F}(z) \geq \operatorname{dist}[z, \operatorname{Spec}(\mathbb{A})] .
\end{gathered}
$$

Assume that $z$ not in $\operatorname{Spec}(\mathbb{A})$, since $\mathcal{L} \subset \operatorname{Dom}(\mathbb{A})$ then:

$$
\begin{aligned}
\mathbb{F}(z) & =\min _{0 \neq v \in \mathcal{H}} \frac{\|(z-\mathbb{A}) v\|}{\|v\|} \\
& \geq \sup _{0 \neq v \in \mathcal{H}} \frac{\left\|(z-\mathbb{A})^{-1} u\right\|^{-1}}{\|u\|} \\
& =\left\|(z-\mathbb{A})^{-1}\right\|^{-1}=\operatorname{dist}[z, \operatorname{Spec}(\mathbb{A})] \quad \text { so } z \in \mathbb{C}
\end{aligned}
$$

Therefore $\mathbb{F}(x)$ can be small only when $x \in \mathbb{R}$ is close to $\operatorname{Spec}(\mathbb{A})$, so we can make a connection between $\operatorname{Spec}(\mathbb{A})$ and $\mathbb{F}(x)$.

Suppose $[a, b]$ be an interval of the spectrum of $\mathbb{A}$ which lies in it, and

$$
\mathcal{L} \subset \operatorname{Dom}\left(\mathbb{A}^{2}\right)=\{u \in \operatorname{Dom}(\mathbb{A}): \operatorname{Lu} \in \operatorname{Dom}(\mathbb{A})\} .
$$

let $\left[a=z_{0}, z_{1}, z_{2}, \cdots, z_{n}=b\right], \forall z \in \mathbb{R}$,

$$
\begin{aligned}
\mathbb{F}\left(z^{2}\right) & =\min _{0 \neq v \in \mathcal{L}} \frac{\left\langle(z-\mathbb{A})^{2} v, v\right\rangle}{\left\|v^{2}\right\|} \\
& =\min _{0 \neq v \in \mathcal{L}} \frac{\left\|(z-\mathbb{A})^{2} v\right\|}{\|v\|} \\
& =\min _{v \in \mathbb{C}^{n}} \frac{\|Q(v)\|}{\|v\|} \\
& =G(z), v \text { is nonzero }
\end{aligned}
$$

Clearly, $\operatorname{Spec}_{2}(\mathbb{A}, \mathcal{L})=\{z \in \mathbb{C}: G(z)=0\}$. For $z \in \mathbb{C}$, let $Q(z)$ be as in (2.1), $v \neq 0$ :

$$
G(z)=\min _{v \in \mathcal{L}} \frac{\|Q(v)\|}{\|v\|}
$$

Thus the zeros of $G(z)$ appear in conjugate pair.

THEOREM 2.1: (Shargorodsky):

For $\lambda \in \operatorname{Spec}_{2}(\mathbb{A}, \mathcal{L})$, and $G(\lambda)=0$, let

$\lambda_{\text {upp }}=\operatorname{Re}(\lambda)+|\operatorname{Im}(\lambda)|, \quad \lambda_{\text {low }}=\operatorname{Re}(\lambda)-|\operatorname{Im}(\lambda)|$ 
Then:

$$
\left[\lambda_{\text {low }}, \lambda_{\text {upp }}\right] \cap \operatorname{Spec}(\mathbb{A}) \neq \varphi .
$$

Let $\lambda=a+\imath b$ for $\alpha, \beta \in \mathbb{R}$, then:

$$
((\lambda-\mathbb{A}) u,(\bar{\lambda}-\mathbb{A}) v)=((\alpha-\mathbb{A}) u,(\alpha-\mathbb{A}) v)+2 \imath \beta((\alpha-\mathbb{A}) u, v)-\beta^{2}(u, v)
$$

Then either $\beta=0, u \in \mathcal{L}, u=v$ we get:

$$
\begin{array}{r}
\|(\alpha-\mathbb{A}) u\|^{2}-\beta^{2}\|u\|^{2}+2 \imath \beta((\alpha-\mathbb{A}) u, u)=0 \\
\text { So: } \beta^{2}=\frac{\|(a-\mathbb{A}) u\|^{2}}{\|u\|^{2}} \text { and }((\alpha-\mathbb{A}) u, u)=0 \\
\text { So: }|\beta|=\frac{\|(a-\mathbb{A}) u\|}{\|u\|} \geq \mathbb{F}(\alpha) \geq \operatorname{dist}[\alpha, \operatorname{Spec}(\mathbb{A})]
\end{array}
$$

\section{The Boulton and Strauss Method}

The method of second order relative spectra has been shown to reliably approximate the discrete spectrum for a self-adjoint operator in [2]. Boulton and Strauss extended this method to normal operators and find optimal convergence rates for eigenvalues and estimated that by an order of magnitude in [3] [4]. The spectrum of $\mathbb{A}, \operatorname{Spec}(\mathbb{A})$, may be expressed as the union of the discrete spectrum consisting of all isolated eigenvalues of finite multiplicity, $\operatorname{Spec}_{\text {disc }}(\mathbb{A})$, and the essential spectrum, where.

$$
\operatorname{Spec}_{\text {ess }}(\mathbb{A})=\frac{\operatorname{spec}(\mathbb{A})}{\operatorname{Spec}_{\text {disc }}(\mathbb{A})}
$$

In most standard situations the essential spectrum can be found analytically, but points in $\operatorname{Spec}_{\text {disc }}(\mathbb{A})$ are usually estimated by numerical procedures. The standard numerical techniques, such as the Quadratic method, aim at solving Galerkin approximate problems posed in weak form: find $u \in \mathcal{L}-\{0\}$ and $\lambda \in \mathbb{R}$ such that $\langle\mathbb{A} u, v\rangle=\lambda\langle u, v\rangle \quad \forall v \in \mathcal{L} \quad(\mathcal{L}$ is finite dimensional). Boulton and Strauss method depends on sub-interval around the eigenvalues in $\operatorname{Spec}(\mathbb{A})$ to find more accurate approximation enclosure eigenvalues than the Quadratic method around $\lambda$. Let:

$$
\begin{gathered}
\lambda_{\min (\mathbb{A})}=\inf [\operatorname{Spec}(\mathbb{A})] \text { and } \lambda_{\max (\mathbb{A})}=\sup [\operatorname{Spec}(\mathbb{A})] . \\
\forall \mathcal{L} \subset \operatorname{Dom}(\mathbb{A}) ; \operatorname{Spec}_{2}(\mathbb{A}, \mathcal{L}) \subset D\left[\lambda_{\min (\mathbb{A})}, \lambda_{\max (\mathbb{A})}\right] . \\
\lim _{n \rightarrow \infty}\left[\operatorname{Spec}_{2}\left(\mathbb{A}, \mathcal{L}_{n}\right)\right] \subseteq \mathcal{D}\left[\lambda_{\min (\mathbb{A})}, \lambda_{\max (\mathbb{A})}\right] \text { for any sequence }\left(\mathcal{L}_{n}\right) .
\end{gathered}
$$

By corresponding limit sets which has been stimulated by the following property: if $(a, b) \cap \operatorname{Spec}(\mathbb{A})=\varphi$, then:

$$
\operatorname{Spec}_{2}(\mathbb{A}, \mathcal{L}) \cap D(a, b)=\varphi, \forall \mathcal{L} \subset \operatorname{Dom}(\mathbb{A}) .
$$

whenever $z \in \operatorname{Spec}_{2}(\mathbb{A}, \mathcal{L})$.

Thus inclusions of points in the spectrum of $\mathbb{A}$ are achieved from $\operatorname{Re}(z)$ with 
a two-sided explicit residual given by $|\operatorname{Im}(z)|$. Indeed, if $(a, b) \cap \operatorname{Spec}(\mathbb{A})=\lambda$ and $z \in \operatorname{Spec}_{2}(\mathbb{A}, \mathcal{L})$ with $z \in D(a, b)$, then:

$$
\left[\operatorname{Re}(z)-\frac{|\operatorname{Im}(z)|^{2}}{b-\operatorname{Re}(z)}, \operatorname{Re}(z)+\frac{|\operatorname{Im}(z)|^{2}}{\operatorname{Re}(z)-a}\right] \cap \operatorname{Spec}(\mathbb{A})=\{\lambda\}
$$

\section{New Technique to Get More Accurate Results}

we described a new technique to get more accurate bounds for the eigenvalues in $\operatorname{Spec}(\mathbb{A})$. Hobiny was discussed the Quadratic method to estimate enclosure eigenvalues for self-adjoint operator and illustrated the results on the harmonic and anharmonic oscillator models to know the accuracy and efficiency for this method in [5]. We discussed also the bounds of the size of the enclosure eigenvalues and studied them in the context of one dimensional Schrodinger operators, and illustrated that the conjugate pairs of the eigenvalues in $\operatorname{Spec}_{2}(\mathbb{A}, \mathcal{L})$ are closed to the real line, so the conjugate pairs will give small intervals $(a, b) s$ enclosing points in $\operatorname{Spec}_{2}(\mathbb{A})$.

After that Boulton in [9] [10] [11], was modified this studying and gave a value for each $a, b$ within a specific domain for the first five eigenvalues in $\operatorname{Spec}(\mathbb{A})$, (i.e.: $a_{1}<\lambda_{1}<b_{1}, a_{2}<\lambda_{2}<b_{2}, a_{3}<\lambda_{3}<b_{3}, a_{4}<\lambda_{4}<b_{4}, a_{5}<\lambda_{5}<b_{5}$ ).

Our improvement depends on domain expansion around the first five eigenvalues in $\operatorname{Spec}(\mathbb{A})$, we will take a value for a less than $\lambda_{1}$ and a value for $b$ greater than $\lambda_{5}$ and calculate the conjugate pairs of eigenvalues $\left(\lambda_{1}, \lambda_{2}, \lambda_{3}, \lambda_{4}, \lambda_{5}\right)$ (i.e.; around $\lambda_{1}$ we will choose $a_{1}<\lambda_{1}<\lambda_{2}<\lambda_{3}<\lambda_{4}<\lambda_{5}<b_{1}$ where:

$$
\begin{aligned}
& \left(\lambda_{1}\right)_{\text {low }}=\operatorname{Re}(z)-\frac{|\operatorname{Im}(z)|^{2}}{b_{1}-\operatorname{Re}(z)} \\
& \left(\lambda_{1}\right)_{\text {upp }}=\operatorname{Re}(z)+\frac{|\operatorname{Im}(z)|^{2}}{\operatorname{Re}(z)-a_{1}}
\end{aligned}
$$

And the same technique for other eigenvalues. Then we will apply $a, b$ in our programs to find the first five enclosure eigenvalues and produce our comparison by numerical experiments on the harmonic and anharmonic oscillator models.

\section{Schrodinger Operator}

Consider the trial subspace $\mathcal{L}$ constructed via the finite element method on finite segment.

Let $\mathcal{L}>0$ and set $\mathbb{A}=\mathcal{H}$ then, the general Schrodinger equation is:

$$
\mathcal{H}_{\mathcal{L}} u(x)=-u^{\prime \prime}(x)+V(x) u(x) ; x \in(-\mathcal{L}, \mathcal{L})
$$

where $V(x)$ is called the potential function, and it must be bounded below $u(\mathcal{L})=u(-\mathcal{L})=0$; which is called the Dirichlet Boundary Conditions.

If we take $u(x)$ as a common factor then:

$$
\left[\frac{-\mathrm{d}^{2}}{\mathrm{~d} x^{2}}+V(x)\right] u(x)=\lambda u(x)
$$


Take:

$$
\mathcal{H}=\frac{-\mathrm{d}^{2}}{\mathrm{~d} x^{2}}+V(x) \text { then } \mathcal{H} u(x)=\lambda u(x)
$$

so the operator $\mathcal{H}$ is a differential equation.

$$
\text { If } V(x)=x^{2}
$$

then the equation is related to harmonic oscillator model.

$$
\text { If } V(x)=x^{4}
$$

then the equation is related to anharmonic oscillator model.

We compare between the Quadratic method, Boulton \& Strauss and our development on these models to calculate the enclosure eigenvalues to know which one is the best in this field.

\section{Harmonic Oscillator Model}

The Harmonic Oscillator is one of the most important models of quantum theory.

Let $\mathcal{H}^{\text {har }}=\mathbb{A}$, for $V(x)=x^{2}$, then the exact eigenvalue is $\lambda_{j+1}=2 j+1$; $j \in \mathbb{N}$.

By Schrodinger equation we have:

$$
\begin{aligned}
& \mathcal{H}^{\text {har }}(x)=-u^{\prime \prime}(x)+x^{2} u(x) \\
& \text { And } \mathcal{H}^{\text {har }}(x)=\lambda u(x)
\end{aligned}
$$

This equation can be solved explicitly and we can find the approximation eigenvalues using Matlab program by matrices $M, N, R$ where,

$$
\begin{gathered}
M=\left[\left(\Psi^{\prime}, \Psi^{\prime}\right)\right] \\
N=\left[x^{2} \Psi, \Psi\right] \\
R=[(\Psi, \Psi)]
\end{gathered}
$$

And $\lambda_{\text {app }}=\frac{M+N}{R}$.

Now we compute $\operatorname{Spec}_{2}\left(\mathcal{H}_{6}^{\text {har }}, L_{6}^{\text {har }}\right)$ as described before and calculate the eigenvalues enclosure. All the coefficients of the matrices were found analytically.

NOTE: In our improvement method we choose $(n=200, L=6)$ to compare our results with Boulton and Strauss method, which approximate the first five eigenvalues of harmonic and anharmonic models with $n=200, L=6$.

Figure 1 and Figure 2 show the conjugate pair for each eigenvalue with upper and lower bounds of eigenvalues in $\operatorname{Spec}_{2}\left(\mathcal{H}_{6}^{\text {har }}\right)$.

Trial 1: In this trial we use the Quadratic method, Boulton \& Strauss method, and our improvement with: $n=200, L=6$, to find the first five approximation enclosure eigenvalues (upper and lower eigenvalues) of $\mathcal{H}_{6}^{\text {har }}$, where $h=\frac{2 L}{n}$ (see Table 1, Figure 3). 
We calculate the Error between the exact and the lower bound for the first five approximation enclosure eigenvalues of $\mathcal{H}_{6}^{\text {har }}$. error $=$ exact $_{j}-\lambda_{j}$. The slope of the graphs is close to the value (6) in all cases as $n$ increases (Figure 3).

Table 1. Approximating enclosures for the first five eigenvalues of $\mathcal{H}_{6}^{\text {har }}$ with $n=$ $200, \lambda_{\text {low }}$ is the lower bound of the enclosing $\lambda$ and $\lambda_{\text {upp }}$ is the upper bound.

\begin{tabular}{|c|c|c|c|c|}
\hline \multicolumn{2}{|c|}{$\Lambda$} & Quadratic method & Boulton and Straus method & Our improvement method \\
\hline \multirow{2}{*}{$\lambda_{1}$} & $U p p$ & 1.000343714729056 & 1.000000236289758 & 1.000000118025628 \\
\hline & low & 0.999656285291227 & 0.999999763730525 & 0.9999999868997263 \\
\hline \multirow{2}{*}{$\lambda_{2}$} & $U p p$ & 3.001030615492415 & 3.000002124427357 & 3.000000212522912 \\
\hline & low & 2.998969384689878 & 2.999997875754936 & 2.999999882073825 \\
\hline \multirow{2}{*}{$\lambda_{3}$} & $U p p$ & 5.002199221800688 & 5.000009673565887 & 5.000000537815785 \\
\hline & low & 4.997800779032315 & 4.999990327267100 & 4.999999463031893 \\
\hline \multirow{2}{*}{$\lambda_{4}$} & $U p p$ & 7.003900035336483 & 7.000030421847483 & 7.000001171500465 \\
\hline & low & 6.996099967297225 & 6.999969580786066 & 6.999998311445117 \\
\hline \multirow{2}{*}{$\lambda_{5}$} & $U_{p p}$ & 9.006150673512703 & 9.000075664791041 & 9.000001996954438 \\
\hline & low & 8.993849333094289 & 8.999924341814951 & 8.999996566707424 \\
\hline
\end{tabular}

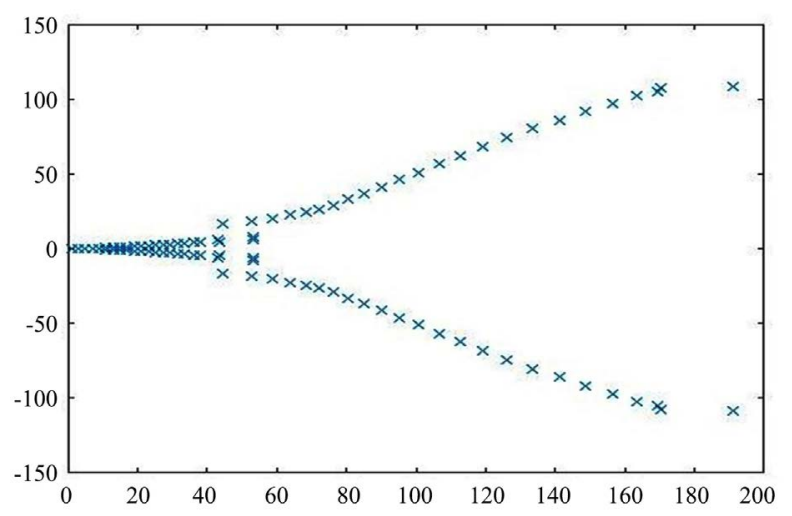

Figure 1. Second order spectra relative to $L^{\text {har }}$. The horizontal axis is the real part of the points in $\operatorname{Spec}_{2}\left(\mathcal{H}_{6}^{\text {har }}, L_{6}^{\text {har }}\right)$ and the vertical axis is imaginary part.

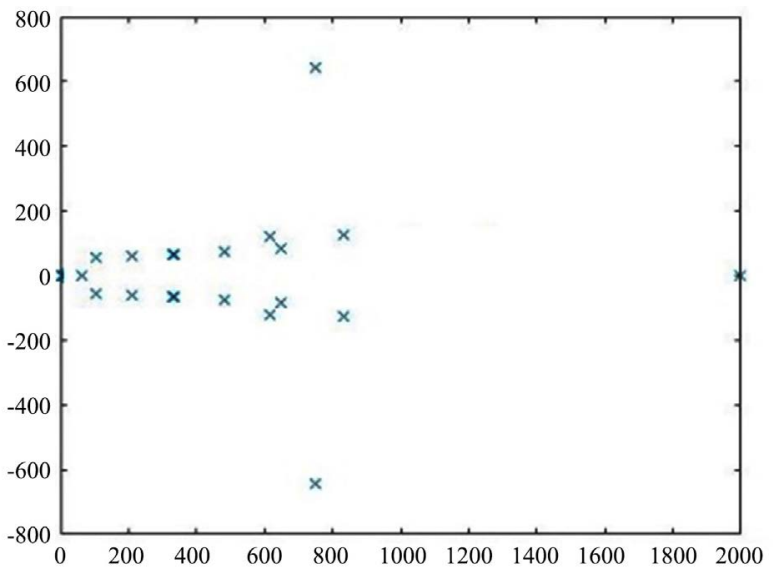

Figure 2. $\operatorname{Spec}_{2}\left(\mathcal{H}_{6}^{\text {har }}\right)$ and illustration on the end pints of the segment given in Theorem 2.1, $\operatorname{Re}(z)-|\operatorname{Im}(z)|$ and $\operatorname{Re}(z)+|\operatorname{Im}(z)|$. 


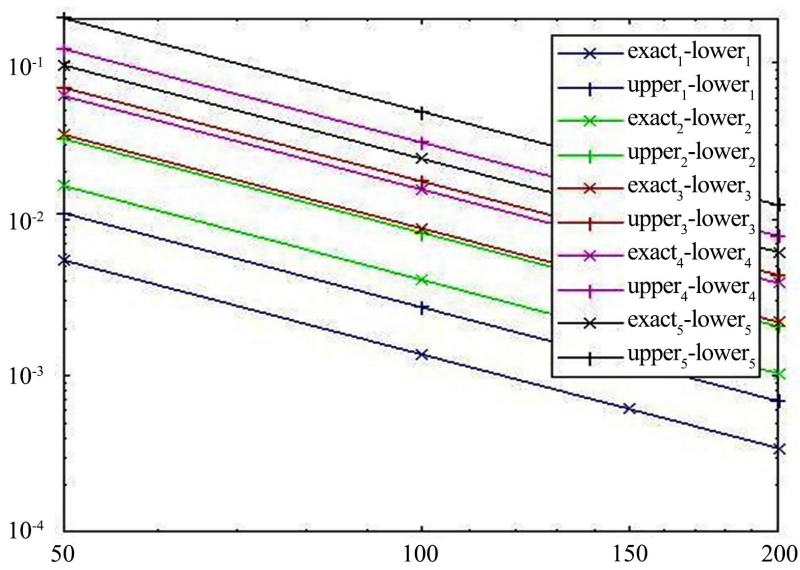

(a)

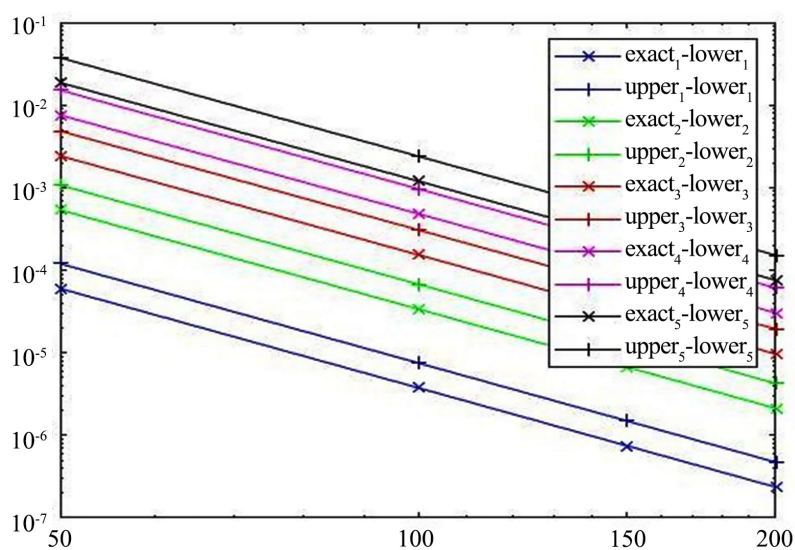

(b)

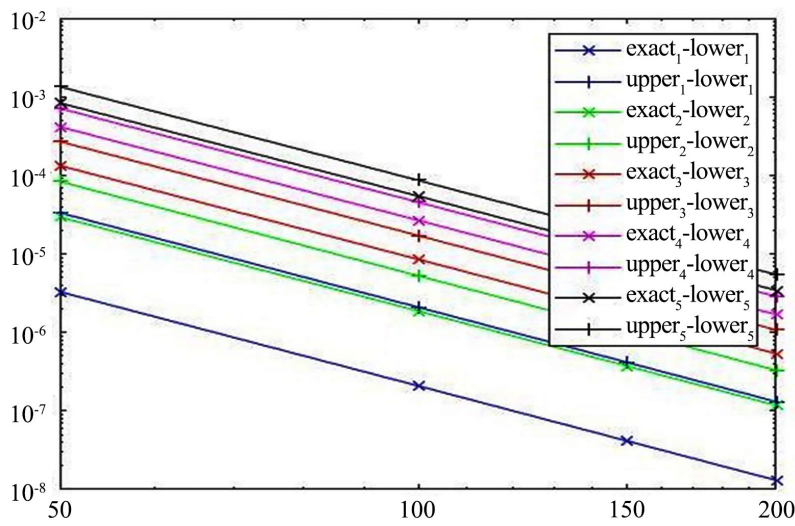

(c)

Figure 3. Approximating enclosures for the first five eigenvalues of $\mathcal{H}_{6}^{\text {har }}$ by the: Quadratic, Boulton \& Strauss, and Our improvement methods with $n=200, \lambda_{\text {low }}$ is the lower bound of the enclosing $\lambda$ and $\lambda_{\text {upp }}$ is the upper bound. (a) This sub-figure shows the error between the exact and lower bound for the first five approximation eigenvalues of $\mathcal{H}_{6}^{\text {har }}$ using the quadratic method; (b) This sub-figure shows the error between the exact and lower bound for the first five approximation eigenvalues of $\mathcal{H}_{6}^{\text {har }}$ using the Boulton \& Strauss method; (c) This sub-figure shows the error between the exact and lower bound for the first five approximation eigenvalues of $\mathcal{H}_{6}^{\text {har }}$ using our improvement method. 


\section{Anharmonic Oscillator Model}

The anharmonic oscillator is another model of Schrodinger equation in one dimension. This model is one of the most important problems of quantum mechanics.

We choose this model to show that the technique for both methods can be applied to operator where the exact spectrum is not known.

Let $\mathcal{H}_{6}^{a n h}=A$, for $V(x)=x^{4}$ then; by Schrodinger equation we have

$$
\begin{aligned}
& \mathcal{H}^{a n h}(x)=-u^{\prime \prime}(x)+x^{4} u(x) \\
& \text { And } \mathcal{H}^{a n h}(x)=\lambda u(x)
\end{aligned}
$$

The exact eigenvalue is unknown.

This equation can be solved explicitly and we can find the approximation eigenvalues using Matlab program by matrices $M, N, R$ where:

$$
\begin{gathered}
M=\left[\left(\Psi^{\prime}, \Psi^{\prime}\right)\right] \\
N=\left[x^{4} \Psi, \Psi\right] \\
R=[(\Psi, \Psi)]
\end{gathered}
$$

We compute $\operatorname{Spec}_{2}\left(\mathcal{H}_{6}^{a n h}, L_{6}^{a n h}\right)$, as in Figure 4.

Trial 2: In this trial we use the Quadratic method, Boulton \& Strauss method, and Our improvement technique with: $n=200, L=6$, to find the first five approximation enclosure eigenvalues (upper and lower eigenvalues) of $\mathcal{H}_{6}^{\text {anh }}$, where

\begin{tabular}{|c|c|c|c|c|}
\hline & $\Lambda$ & $\begin{array}{l}\text { Quadratic } \\
\text { method }\end{array}$ & $\begin{array}{c}\text { Boulton } \\
\text { and } \\
\text { Straus method }\end{array}$ & $\begin{array}{l}\text { Our } \\
\text { improvement } \\
\text { method }\end{array}$ \\
\hline \multirow{2}{*}{$\lambda_{1}$} & $U p p$ & 1.060362557981696 & 1.060362252449401 & 1.061066062252193 \\
\hline & low & 1.060361563013190 & 1.060362061273195 & 1.059658118801031 \\
\hline \multirow{2}{*}{$\lambda_{2}$} & $U p p$ & 3.799676880351021 & 3.799673918715551 & 3.797040801641494 \\
\hline & low & 3.799638443628093 & 3.799672602711578 & 3.797040801641494 \\
\hline \multirow{2}{*}{$\lambda_{3}$} & $U p p$ & 7.455732063205368 & 7.455701633603200 & 7.462745619537849 \\
\hline & low & 7.455606688132128 & 7.455694527185979 & 7.448650264962926 \\
\hline \multirow{2}{*}{$\lambda_{4}$} & $U p p$ & 11.644110613912629 & 11.644757012619969 & 11.659764086317372 \\
\hline & low & 11.644649763222517 & 11.644727274867936 & 11.629726975328792 \\
\hline \multirow{2}{*}{$\lambda_{5}$} & $U p p$ & 16.262159917276765 & 16.261854836128617 & 16.289304652770664 \\
\hline & low & 16.261391679238212 & 16.264709951625957 & 16.234347516123545 \\
\hline
\end{tabular}

$$
h=\frac{2 L}{n}
$$

(see Table 2, Figure 5).

Table 2. Approximating enclosures for the first five eigenvalues of $\mathcal{H}_{6}^{\text {anh }}$ with $n=$ $200, \lambda_{\text {low }}$ is the lower bound of the enclosing $\lambda$ and $\lambda_{\text {upp }}$ is the upper bound. 


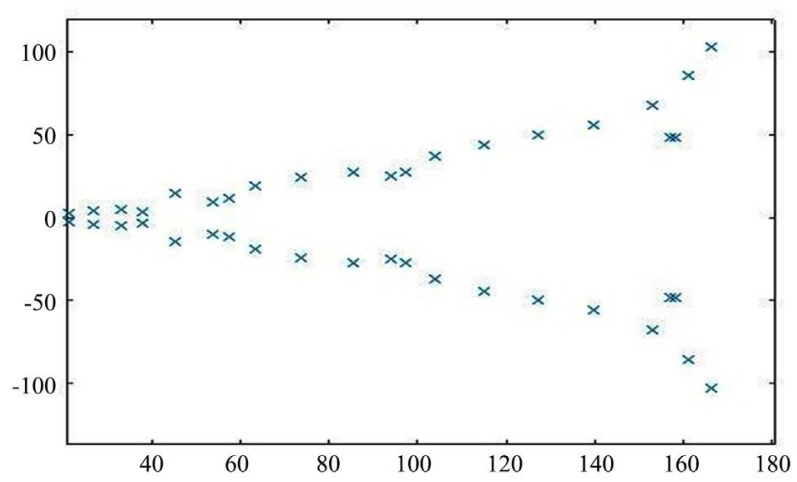

Figure 4. Second order spectra relative to $L_{6}^{a n h}$. The horizontal axis is the real part of the points in $\operatorname{Spec}_{2}\left(\mathcal{H}_{6}^{a n h}, L_{6}^{a n h}\right)$ and the vertical axis is imaginary part.
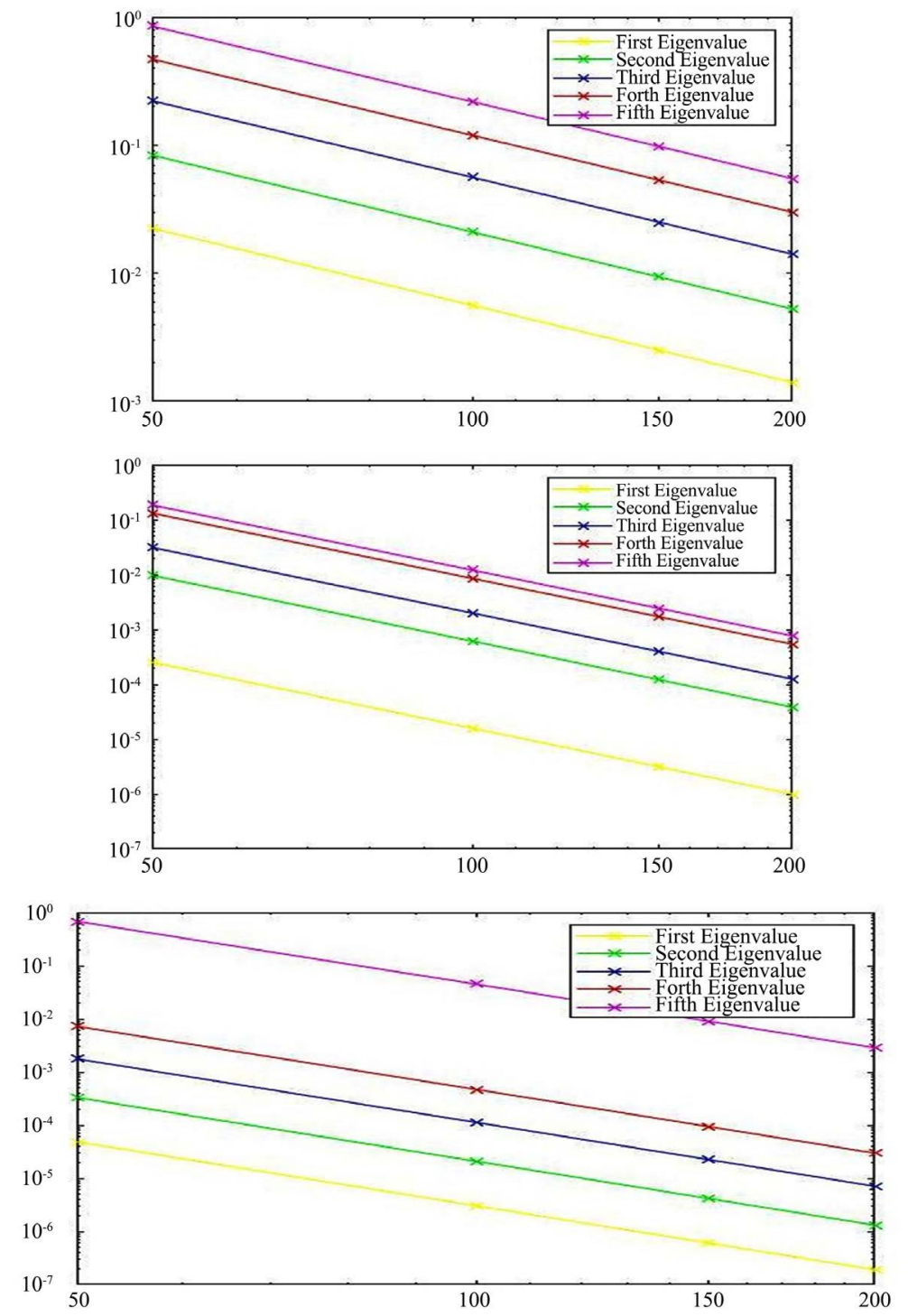

Figure 5. Approximating enclosures for the first five eigenvalues of $\mathcal{H}_{6}^{\text {anh }}$ by the Quadratic, Boulton \& Strauss, and Our improvement methods with $n=200, \lambda_{\text {low }}$ is the lower bound of the enclosing $\lambda$ and $\lambda_{\text {upp }}$ is the upper bound. 


\section{Conclusions}

We compute $\operatorname{Spec}_{2}\left(\mathcal{H}_{6}^{\text {har }}, L_{6}^{\text {har }}\right)$ and $\operatorname{Spec}_{2}\left(\mathcal{H}_{6}^{\text {anh }}, L_{6}^{\text {anh }}\right)$, as we described before and calculate the eigenvalues enclosure. All the coefficient of the matrices $\mathbb{A}_{0}$, $\mathbb{A}_{1}$ and $\mathbb{A}_{2}$ were found analytically.

Figure 1 and Figure 4 show $\operatorname{Spec}_{2}\left(\mathcal{H}_{6}^{\text {har }}, L_{6}^{\text {har }}\right)$ and $\operatorname{Spec}_{2}\left(\mathcal{H}_{6}^{\text {anh }}, L_{6}^{\text {anh }}\right)$ for value $n=200$, clearly in both the second-order relative spectra is not the same.

In Table 1 and Table 2 we show the approximation of the first five eigenvalues enclosures of $\mathcal{H}_{6}^{\text {har }}, \mathcal{H}_{6}^{\text {anh }}$ respectively, with $n=200$ by three different methods (Quadratic method, Boulton \& Strauss method, and Our improvement) to compare between these methods and identify which one is more accurate for computing eigenvalues.

For the Harmonic oscillator model, the error between the approximation results and the first five exact eigenvalues $(1,3,5,7,9)$ in our technique is less than the error between the exact and the approximation eigenvalues by the Boulton and Strauss method, and the same thing between of the Quadratic method results and the exact eigenvalues, so the approximation enclosure eigenvalues by our technique is more accurate and effective than the Boulton \& Strauss method and the Quadratic method, also Figure 3 shows that clearly.

For the anharmonic oscillator model, the exact eigenvalues are unknown but by results and Figure 5, we can confirm the previous result.

\section{Conflicts of Interest}

The author declares no conflicts of interest regarding the publication of this paper.

\section{References}

[1] Kato, T. (1949) On the Upper and Lower Bounds of Eigenvalues. Journal of the Physical Society of Japan, 4, 334-339. https://doi.org/10.1143/JPSJ.4.334

[2] Strauss, M. (2013) The Second Order Spectrum and Optimal Convergence. Mathematics of Computation, 82, 2305-2325. https://doi.org/10.1090/S0025-5718-2013-02693-2

[3] Boulton, L. and Strauss, M. (2011) On the Convergence of second order spectra and Multiplicity. Proceedings of the Royal Society A Mathematical Physical and Engineering Sciences, 467, 264-284. https://doi.org/10.1098/rspa.2010.0233

[4] Boulton, L. and Strauss, M. (2007) Stability of Quadratic Projectione Methods. Operators and Matrices, 17, 217-233. https://doi.org/10.7153/oam-01-15

[5] Boulton, L. and Hobiny, A. (2013) On the Quality of Complementary Bounds for Eigenvalues. Calcolo, 52, 577-601. https://doi.org/10.1007/s10092-014-0131-y

[6] Davies, E.B. and Plum, M. (2004) Spectral Pollution. IMA Journal of Numerical Analysis, 24, 417-438. https://doi.org/10.1093/imanum/24.3.417

[7] Davies, E.B. (1996) Spectral Theory and Differential Operators. Cambridge University Press, Cambridge.

[8] Levitin, M. and Shargorodsky, E. (2004) Spectral Pollution and second order relative Spectra for Self-Adjoint Operators. IMA Journal of Numerical Analysis, 24, 
393-416. https://doi.org/10.1093/imanum/24.3.393

[9] Boulton, L. and Levitin, M. (2007) On Approximation of the Eigenvalue of Perturbed Periodic Schrodinger Operators. Journal of Physics A Mathematical and Theoretical, 40, 9319. https://doi.org/10.1088/1751-8113/40/31/010

[10] Boulton, L. and Hobiny, A. (2016) On the Convergence of the Quadratic Method. IMA Journal of Numerical Analysis, 36, 1310-1333. https://doi.org/10.1093/imanum/drv036

[11] Boulton, L., Garcia del Moral, M.P. and Restuccia, A. (2012) Spectral Properties in Super Symmetric Matrix Models. Nuclear Physics B, 856, 716-747.

https://doi.org/10.1016/j.nuclphysb.2011.11.017 